\title{
Comparative Study of Pulse Shaping Filters in FBMC
}

\author{
Mohammed Kasim Al-Haddad ${ }^{1}$, Hadi T. Ziboon ${ }^{2}$ \\ ${ }^{1}$ Electronics and Communications Engineering Dpt. The University of Baghdad. \\ ${ }^{2}$ Electrical Engineering Engineering Dpt. The University of Technology. \\ mohammed.alhadad@coeng.uobaghdad.edu.iq,haditarishziboon@yahoo.co.uk
}

\begin{abstract}
Filter Bank Multicarrier (FBMC) is a multitone modulation technique that is expected to replace the Orthogonal Frequency Division Multiplexing (OFDM) due to its inherent characteristics that makes it immune to channel dispersive effect on the transmitted signal in both time and frequency. The most effective ingredient in the FBMC is the pulse shaping that the OFDM symbol lacks. In this paper, a comparative study is presented between different pulse shapes used in the FBMC like the RRC, PHYDIAS, IOTA and Hermite function alongside the conventional OFDM.
\end{abstract}

Index Terms - FBMC, shaping filter, ambiguity function, Hermite function, PHYDYAS, IOTA, $R R C, S I R$.

\section{INTRODUCTION}

Mobile communications have been under increasing demand for higher data rates for different services that are mostly voice communications and data communications. This has led to the development of the mobile technologies throughout the different generations of mobile communications up to the $4 \mathrm{G}$ that is introduced by LTE. In addition to that, future communications will support machine-type communication services. This continuous demand for the higher data rate has been addressed by the development of the air interface of different technologies. So far, the multicarrier modulation (MCM) represented by the Cyclic Prefix Orthogonal Frequency Division Multiplexing (CP-OFDM) has proven to be the best type of modulation for wireless communication due to its reduced complexity. Although the CP-OFDM as a member of the MCM family can mitigate the wireless channel impairment namely the multipath fading by subdividing a frequency selective channel into $\mathrm{N}$ flat subchannels that can be equalized by simple one-tap equalizer, it is still has the disadvantages of having the cyclic prefix (CP) that reduces the spectral efficiency in addition to the high Out-of-Band (OOB) spectral leakage that necessitates the use of large guard bands between different CP-OFDM signals that further degrades the spectral efficiency. This OOB spectral leakage is basically because no pulse shaping is used in OFDM, which makes the symbol shape a rectangular window. Researchers have shifted attention towards the Filter Bank Multicarrier (FBMC) modulation which when used alongside with the Offset Quadrature Amplitude modulation (OQAM) results in improved spectral efficiency and spectral shape because the CP is dropped, and due to the use of specially designed shaping filters that significantly reduce the OOB emission. Moreover, research has shown that FBMC is more suitable for time and frequency dispersive channels [1] and multiple access schemes [2]. This makes the FBMC one of the main candidates for future communication technologies targeting the $5 \mathrm{G}$ requirements. In previous works, researches are mostly interested in comparing CP-OFDM with FBMC with certain pulse shaping filter under wireless channel conditions like [1], [2] and [11] where PHYDYAS 
filter is used in FBMC. In [3] a study of the pulse shaping filter for windowed OFDM with a little emphasis in shaping filtered used in FBMC. In [4] an experimental comparison is provided between OFDM and FBMC with RRC filter. In [8] the comparison was between CP-OFDM and FBMC based on EGF and half cosine filters. In [10] various shaping is considered and the performance is evaluated for frequency and timing offset under fixed channel conditions. Works addressing FBMC specific shaping filters are found in [9] for PHYDYAS filter, [13] Hermite function filter and [14] IOTA filter. Three main properties are considered as requirements upon which the FBMC was developed; (i) Orthogonality, which is necessary for the perfect recovery of the transmitted symbols. Orthogonality is already satisfied in OFDM. (ii) locality, which is required for the signal to have high OOB attenuation and to acquire some immunity against the channel dispersive effects. This is not satisfied by OFDM. (iii) ideal spectral efficiency, which is satisfied in the FBMC with the aid of OQAM and also satisfied by the OFDM but not in CP-OFDM. The first and second points are not exactly satisfied $100 \%$ in FBMC but actually very close to the ideal case. The situation varies for different pulse shapes and in this paper, the investigation is made to see how the different pulse shapes compare among each other. It is worth mentioning here that FBMC nomenclature implies two meaning; the FBMC as a multicarrier with shaping filter technique and the OQAM, which is the splitting of the real and imaginary parts of the QAM symbol and transmitting them at double the QAM rate. The combination of both is called FBMC-OQAM but for simplicity, it is often referred to by only FBMC as is the case in this paper.

\section{CONSTRUCTION AND RECONSTRUCTION OF FBMC}

In general, the FBMC signal can be expressed as

$$
s(t)=\sum_{n=-\infty}^{\infty} \sum_{k=0}^{N-1} a_{n, k} \gamma_{n, k}(t)
$$

Where $a_{n, k}$ is real PAM data symbols and $\gamma_{n, k}$ is the basis function given by

$$
\gamma_{n, k}(t)=\phi_{n, k} h\left(t-n \tau_{0}\right) e^{j 2 \pi k v_{0}}
$$

Where $h(t)$ is a real symmetric pulse shaping filter and $\phi_{n, k}$ is a phase factor given by

$$
\phi_{n, k}=e^{j(\pi / 2)((n+k) \bmod 2)}=j^{(n+k) \bmod 2}
$$

The values of $\phi_{n, k}$ can be seen in the manner that when the time index $n$ is even, $\phi_{\text {even }, k}=1, j, 1, j \ldots$ and when $n$ is odd, $\phi_{o d d, k}=1, j, 1, j \ldots$ The values of $\tau_{0}$ and $v_{0}$ are chosen such that $\tau_{0} v_{0}=1 / 2$. In the OFDM case

$$
s(t)=\sum_{n=-\infty}^{\infty} \sum_{k=0}^{N-1} x_{n, k} \operatorname{rect}\left(t-n \tau_{0}\right) e^{j 2 \pi k v_{0} t}
$$

Here the data symbols $x_{n, k}$ are complex QAM symbols and the values of $\tau_{0}=T$ and $v_{0}=1 / T$. The shaping filter is the trivial rectangular pulse. The spectral efficiency for both (2) and (4) is given in symbols/s/Hz by [5] $\eta=1 /\left(\tau_{0} v_{0}\right)$. The OFDM signal in (4) has $\eta=1$ which is the highest possible value of spectral efficiency to keep basis functions $\gamma_{n, k}$ orthogonal. The OFDM signal is not suitable for mobile wireless channels because it cannot combat the multipath effect. Therefore, the cyclic prefix technique is used where $\tau_{0}=T+T_{C P}$ and $v_{0}=1 / T$ in (4), combined with single tap equalizer per subcarrier, this is referred to by CP-OFDM. Although CP-OFDM has improved performance against dispersive channels, it has a lower spectral efficiency than the conventional OFDM, $\eta<1$. In FBMC the use of the shaping filter eliminates the need for the CP. Unfortunately, the transmission of complex symbols cannot be achieved in FBMC because according to Balian-Low theorem [7] that simply states if 
$\tau_{0} \nu_{0}=1$, then the product of the effective duration and effective bandwidth of the signal, discussed in section IV, is infinite which is the case in OFDM. In FBMC, real data are transmitted instead but this would make the spectral efficiency of FBMC half of spectral efficiency of the OFDM because the transmission of a complex symbol in OFDM is equivalent to transmission of two real symbols. This is compensated by making the signaling rate in FBMC twice as fast of that in OFDM with the same frequency separation between subcarriers, i.e., $\tau_{0}=T / 2$ and $v_{0}=1 / T$, i.e., $\eta=2$. The net spectral efficiency in bit/s/Hz will be the same in FBMC and OFDM. The reconstruction of the FBMC data symbols is achieved by

$$
\begin{gathered}
\hat{a}_{n, k}=\left\langle s(t), \gamma_{n, k}(t)\right\rangle=\operatorname{Re}\left\{\int_{-\infty}^{\infty} s(t) \phi_{n, k}^{*} h^{*}\left(t-n \tau_{0}\right) e^{-j 2 \pi k v_{0} t} d t\right\} \\
\hat{a}_{n, k}=\operatorname{Re}\left\{\sum_{l} \sum_{m} \phi_{l, m} \phi_{n, k}^{*} \int_{-\infty}^{\infty} h\left(t-l \tau_{0}\right) h\left(t-n \tau_{0}\right) e^{j 2 \pi(m-k) v_{0} t} d t\right\}
\end{gathered}
$$

Here the real operator $\operatorname{Re}\{\}$ is used in the definition of the inner product $<\cdot,>$. It can be shown that when $h(t)$ is real and symmetric, the expression inside the $\operatorname{Re}\{\}$ operator is purely imaginary in the case if both pairs $(l, m)$ and $(n, k)$ have unequal parity, that is $(l, m) \bmod 2 \neq(n, k) \bmod 2$, therefore, the real operator will produce zero value. When $(l, m)$ and $(n, k)$ have the same parity, the expression inside the $\operatorname{Re}\{\}$ operator will be real and (5) will be reduced to [5]

$$
\hat{a}_{n, k}=\sum_{l} \sum_{m} a_{l, m} A_{h}\left(2(l-n) \tau_{0}, 2(m-k) v_{0}\right)
$$

Where $A_{h}(\tau, v)$ is the auto-ambiguity function of $h(t)$.

$$
A_{h}(\tau, v)=\int_{-\infty}^{\infty} h(t+\tau / 2) h(t-\tau / 2) e^{j 2 \pi v t} d t
$$

Perfect recovery of the symbol $a_{n, k}$ is achieved if

$$
A_{h}\left(2 l \tau_{0}, 2 m v_{0}\right)= \begin{cases}1 & (l, m)=(0,0) \\ 0 & (l, m) \neq(0,0)\end{cases}
$$

The condition in (8) is the orthogonality condition mentioned earlier that $h(t)$ needs to satisfy. A convenient way to demonstrate the orthogonality condition in FBMC is through the lattice shown in Fig. 1 where the basis functions $\gamma_{n, k}$ are shown as lattice points distributed along the time and frequency axes. The lattice is divided into four sub-lattices according to the values of $l$ and $m$ : $\mathbf{E E}$, EO, OE, and $\mathbf{O O}$ where $\mathbf{E}$ stands for even and $\mathbf{O}$ stands for odd. Whenever the values of the pairs $(l, m)$ and $(n, k)$ fall in two different sub-lattices, the inner product as in (6) produces imaginary value inside the $\operatorname{Re}\{\}$ operator, hence the outcome is zero, and when the pairs $(l, m)$ and $(n, k)$ fall in the same sub-lattice, it is up to the design of $h(t)$ to satisfy (8). In practice, the orthogonality is not ideally satisfied according to (8) but it is satisfied within acceptable limits. 


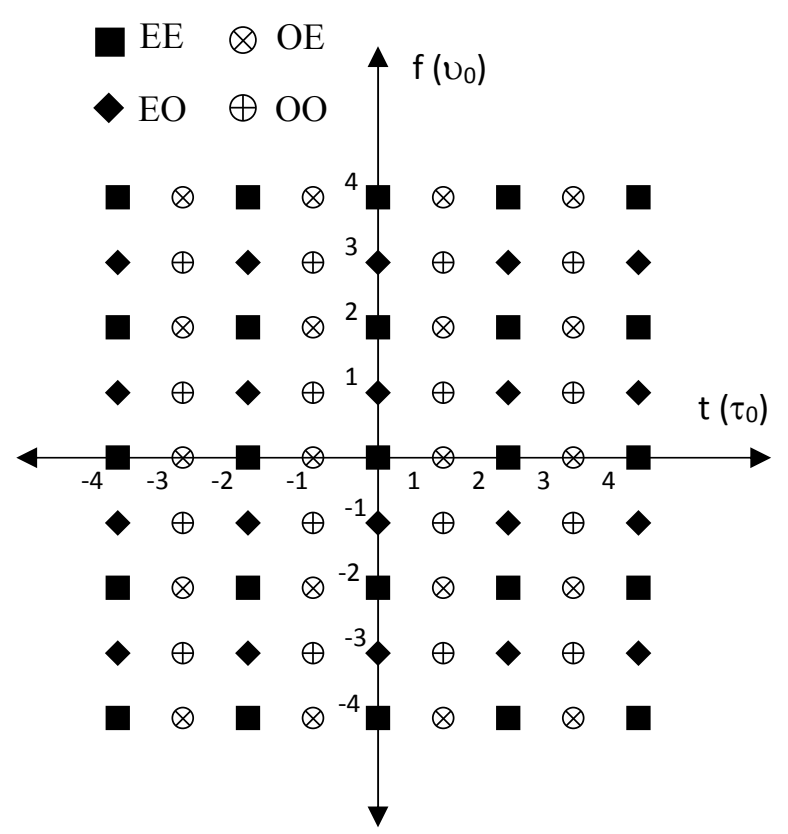

FIG. 1. LATTICE POINTS OF FBMC SIGNAL.

\section{THE EFFECT OF THE CHANNEL}

One of the main goals of the FBMC is to reduce the dispersive effect of the channel in time and frequency that appears as intersymbol interference (ISI) and intercarrier interference (ICI) and hence increasing the signal-to-interference ratio (SIR). When passing the FBMC signal through a timevarying channel $\mathrm{c}(t, \tau)$ the received signal will be

$$
r(t)=\int c(t, \tau) s(t-\tau) d \tau
$$

By plugging (1) in (9) we get

$$
\begin{gathered}
r(t)=\sum_{n} \sum_{k} x_{n, k} g_{n, k}(t) \\
g_{n, k}(t)=e^{j 2 \pi k v_{0} t} \int c(t, \tau) h\left(t-\tau-n \tau_{0}\right) d \tau
\end{gathered}
$$

Where $x_{n, k}=a_{n, k} \phi_{n, k}$. Assuming the only $a_{0,0}$ is transmitted, from (6) the recovered symbol will be

$$
\begin{gathered}
\hat{a}_{0,0}=<\gamma_{0,0}(t), r(t)>=\operatorname{Re}\left\{\sum_{n} \sum_{k} x_{n, k} \int h(t) g_{n, k}^{*}(t) d t\right\} \\
\hat{a}_{0,0}=\operatorname{Re}\left\{\sum_{n} \sum_{k} x_{n, k} \int h(t) g^{*}\left(t-n \tau_{0}\right) e^{-j 2 \pi v_{0} t} d t\right\}
\end{gathered}
$$

Where $g(t)=g_{00}(t)$

$$
\hat{a}_{0,0}=\operatorname{Re}\left\{\sum_{n} \sum_{k} x_{n, k} A_{h g}\left(n \tau_{0}, k v_{0}\right)\right\}
$$

Where $A_{h g}(\tau, \nu)$ is the cross ambiguity function defined by

$$
A_{h g}(\tau, v)=\int h(t) g^{*}(t-\tau) e^{-j 2 \pi t} d t
$$

By considering only the points of the lattice that $a_{0,0}$ belong

$$
\hat{a}_{0,0}=\sum_{n} \sum_{k} a_{2 n, 2 k} A_{h g}\left(2 n \tau_{0}, 2 k v_{0}\right)
$$




$$
=a_{0,0} A_{h g}(0,0)+\sum_{(n, k) \neq(0,0)} a_{2 n, 2 k} A_{h g}\left(2 n \tau_{0}, 2 k v_{0}\right)=S+I
$$

Where $S$ is the received symbol, $I$ is the interference signal, from which the average received signal power $\sigma_{S}^{2}$ and average interference power $\sigma_{I}^{2}$ can be obtained.

$$
\begin{gathered}
\sigma_{S}^{2}=E\left\{\left.S\right|^{2}\right\}=E\left\{\left.a_{0,0} A_{h g}(0,0)\right|^{2}\right\} \\
\sigma_{I}^{2}=E\left\{\left.I\right|^{2}\right\}=E\left\{\sum_{(n, k) \neq(0,0)}\left|a_{n, k} A_{h g}\left(2 n \tau_{0}, 2 k v_{0}\right)\right|^{2}\right\}
\end{gathered}
$$

In (19), the triangle inequality has been used to account for the worst case of the interference signal. From the above equations, we can find the signal-to-interference ratio $S I R=\sigma_{S}^{2} / \sigma_{I}^{2}$ by simply passing the shaping filter through the channel and finding the cross ambiguity function between the input and the output of the channel. The channel will have a smearing effect on the ambiguity function such that the null points of the ambiguity function will no longer be zero, and these nonzero values will add up as an interference signal to the recovered data symbol. This cross-ambiguity function is shown in Fig. 2.

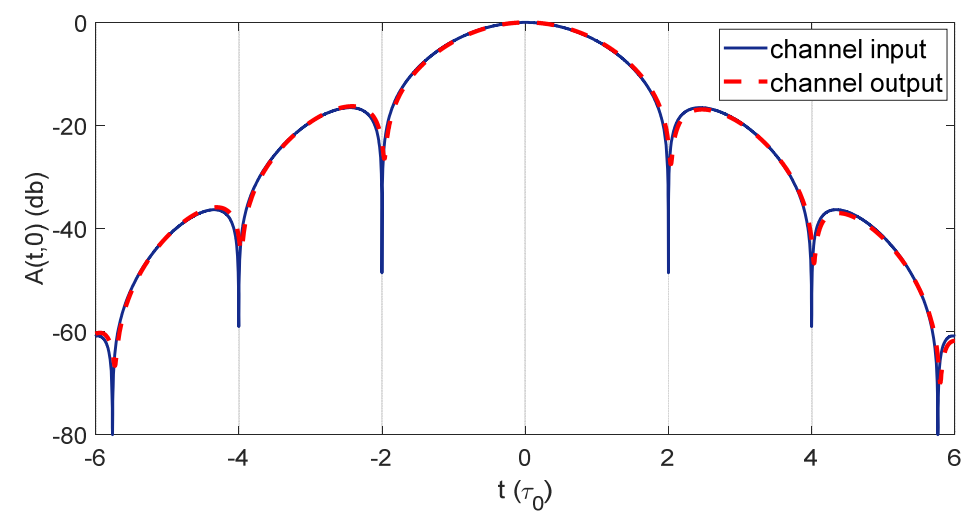

FIG. 2. THE CHANNEL EFFECT ON THE AMBIGUITY FUNCTION OF FBMC SIGNAL.

\section{SIGNAL LOCALIZATION}

It has been mentioned earlier that one of the desired features in FBMC is signal locality. This feature helps in overcoming the channel dispersive effect without the need for the addition of cyclic prefix as in the CP-OFDM case. Signal locality is defined in terms of its effective duration and effective bandwidth, each is defined as [17]

$$
T_{e}^{2}=\frac{1}{E_{s}} \int_{-\infty}^{\infty} t^{2}|h(t)|^{2} d t, \quad B_{e}^{2}=\frac{1}{E_{s}} \int_{-\infty}^{\infty} f^{2}|H(f)|^{2} d f
$$

Where $h(t)$ is a unit energy signal and $H(f)$ is its Fourier transform. For a Gaussian signal given by

$$
h_{g}(t)=2^{1 / 4} e^{-\pi t^{2}}
$$

It has an isotropic Fourier transform given $H_{g}(f)=h_{g}(f)$. The effective time and bandwidth can be obtained as $T_{e}=B_{e}=1 / 2 \sqrt{\pi}$, the product will be $T_{e} B_{e}=1 / 4 \pi$. This is a lower bound for the product $T_{e} B_{e}$ which is an indication of the signal good localization. Any other signal will have a higher value than $1 / 4 \pi$. If we use $1 / 4 \pi$ as a normalization factor, we can define the localization factor as

$$
\lambda=4 \pi T_{e} B_{e} \geq 1
$$

The equality holds for the Gaussian signal, which is although ideal in terms of the localization factor, it is not orthogonal, hence it is not suitable for FBMC as a shaping filter. In some references [10], the localization parameter is defined by $1 / 4 \pi T_{e} B_{e}$ and is called the 
Heisenberg uncertainty parameter, but we prefer the definition as in (22) because it depicts how the signal is spread or confined in both time or frequency by how small or large the value of $\lambda$

\section{SHAPING FILTERS}

In this section, some of the main types of shaping filters that are suitable for FBMC are briefly explained

\section{A. ROOT RAISED COSINE}

Root Raised Cosine (RRC) is a typical Nyquist filter that is bandlimited and exhibits orthogonality in time and frequency; however, it has an infinite time duration. RRC is given by [12]

$$
h_{r r c}(t)=\frac{4 \beta}{\pi T} \cdot \frac{\cos ((1+\beta) \pi t / T)+\sin ((1-\beta) \pi t / T) /(4 \beta t / T)}{1-(4 \beta / T)^{2}}
$$

Where $\beta$ is the roll-off factor whose value is chosen $\beta=1$, and $T$ is the design signaling interval. Since RRC has infinite time duration, it should be truncated up to the desired duration

\section{B. PHYDYAS}

PHYsical layer for DYnamic AccesS and cognitive radio abbreviated as PHYDYAS is a project that proposes the FBMC as the physical layer for dynamic access spectrum management (DASM) and cognitive radio communications. The proposed PHYDYAS filter is given by [9]

$$
h_{P}(t)=1+2 \sum_{k=1}^{K-1} b_{k} \cos (2 \pi k / K T)
$$

Where $1 / T$ is the subcarrier frequency separation and the coefficients $b_{k}$ satisfy the relation $b_{k}^{2}+b_{K-k}^{2}=1$ so that the filter satisfies Nyquist condition. For $K=4$ the coefficients are $b_{1}=0.97196, b_{2}=1 / \sqrt{2}$, and $b_{3}=\sqrt{1-b_{1}^{2}}$. For other values of $K$, the reader may refer to [9]. It can be seen from (24) that $h_{p}(t)$ is periodic with period $K T$ and this period is sampled to $L=K M$ samples. The PHYDYAS filter has a special feature regarding its implementation which is that the shaping can be made in the frequency domain i.e., before the IFFT operation by convolving the set of coefficients $b_{k}$ with the modulating symbols and then applying to the IFFT. However, that would require $K M$ points IFFT [9] instead of $M$ point IFFT in case of the polyphase network (PPN) implementation for the shaping filter as is the case for the other types of shaping filters [15].

\section{Hermite Function}

The Hermite function is a family of functions that are invariant under Fourier transform, meaning that they preserve the same shape in time and frequency domain just like the Gaussian function. In fact, the Gaussian function is considered a zeroth order Hermite function. This property makes the Hermite function to have a good localization [13]. In addition, the different orders of the Hermite functions are orthogonal to each other. These properties make the Hermite functions excellent choice to design a shaping filter. The Hermite function is given by

$$
\psi_{n}(t)=H_{n}(\sqrt{2 \pi} t) e^{-\pi t^{2}}
$$


Where $H_{n}(x)$ is the Hermite polynomial of order $n$ and it can be obtained from the recursive formula: $H_{0}(x)=1, H_{n+1}(x)=2 x H_{n}(x)-H_{n}^{\prime}(x)$ where $H_{n}^{\prime}(x)$ is the derivative of $H_{n}(x)$ with respect to $x$. The Fourier transform of the Hermite function is given by

$$
\int_{-\infty}^{\infty} \psi_{n}(t) e^{-j 2 \pi f f} d t=(-j)^{n} \psi_{n}(f)
$$

Therefore, for orders, multiple of 4 the Hermite function and its Fourier transform are exactly the same. The Hermite filter was designed as the linear combination of Hermite functions with order multiple of 4 . The coefficients are chosen such that the orthogonality is satisfied in the lattice points near and around $(n, k)=(0,0)$ only because the far lattice point have an insignificant contribution as interference and hence having a near perfect (NP) orthogonality

$$
h_{H}(t)=\sum_{k=0}^{4} C_{k} \psi_{4 k}(t)
$$

A complete analytical discussion is found in [13]

\section{IOTA}

Isotropic Orthogonal Transform Algorithm (IOTA) is an algorithm that initially takes the Gaussian function and introduces orthogonality in both time and frequency domain. This is achieved by the orthogonality operator [14]

$$
y(t)=O_{\tau_{0}} x(t)=x(t) / \sqrt{\tau_{0} \sum_{k=-\infty}^{\infty} \mid x\left(t-\left.k \tau_{0}\right|^{2}\right.}
$$

The effect of the operator $O_{z 0}$ on $x(t)$ is making $x(t)$ orthogonal in frequency at multiples of $1 / \tau_{0}$, i.e. in terms of the ambiguity function $A_{y}\left(0, k / \tau_{0}\right)=0$ for $k \neq 0$ and $A_{y}(0,0)=1$. Similarly applying the orthogonality operator to a signal in its frequency domain, $Y(f)=O_{\nu 0} X(f)$ produces a signal that is orthogonal in time such that $A_{y}\left(n / v_{0}, 0\right)=0$ for $n \neq 0$ and $A_{y}(0,0)=1$. Applying both operators as below produces the IOTA filter

$$
h_{I}(t)=O_{\tau_{0}} F^{-1} O_{\nu_{0}} F h_{g}(t)
$$

Where $F$ and $F^{-1}$ are the Fourier transform and inverse Fourier transform.

\section{SIMULATION RESULTS}

A comparison of the above 4 types of shaping filters in addition to the conventional OFDM has been performed under channel effect. Each filter is realized in discrete time as

$$
h(m)=h\left((m-L / 2) t_{s}\right)
$$

Where $m$ is the sample time index, $L$ is the filter length in samples and it is chosen to be $L=1024$ samples and $t_{s}$ is the sampling time which is chosen equals to $32.5 \mathrm{~ns}$, the same as the sampling time in LTE. The $L / 2$ shift is to make the filter a causal filter. The overlapping factor $K=4$. It is important to note that the time interval $T$ that appears in (23) and (24) corresponds to the signaling interval and the symbol duration of the OFDM in seconds. While for the FBMC $T=2 \tau_{0}$ where $\tau_{0}$ is the signaling interval in FBMC and the symbol duration is $K T=L t_{s}$ in seconds. The frequency separation between successive subcarriers in FBMC $v_{0}=1 / T$ which is the same as that in OFDM. Before the evaluation of the channel effect, the localization factor $\lambda$ and the interference power $\sigma_{I}^{2}$ was evaluated for each shaping filter without channel effect using $L=4096$ points. The results are shown in Table I. 
TABLE I. LOCALIZATION FACTOR AND INTRINSIC INTERFERENCE POWER

\begin{tabular}{cccccc}
\hline & Rect. & RRC & PHYDYAS & Hermite & IOTA \\
\hline$T_{e}$ & 0.2887 & 0.2435 & 0.2745 & 0.2015 & 0.2729 \\
$B_{e}$ & 8.4803 & 0.3612 & 0.3280 & 0.4031 & 0.3242 \\
$\lambda$ & 30.7784 & 1.1053 & 1.1314 & 1.0208 & 1.1118 \\
$\sigma_{I}^{2}$ & $8.88 \times 10^{-16}$ & $1.13 \times 10^{-5}$ & $1.17 \times 10^{-7}$ & $2.35 \times 10^{-9}$ & $2.33 \times 10^{-4}$ \\
\hline
\end{tabular}

Table I provides the metrics that are an initial indication about the shaping filter performance under a dispersive channel. The filters with low values of localization factor and interference power are expected to perform well under the dispersive channel. For the rectangular filter, the theoretical value of $\lambda$ is infinite due to the sinc shape of its spectrum and the finite value appearing in the table is due to the discretization of the filter but it is still much higher than the other filters. On the other hand, the interference power $\sigma_{I}^{2}$ is ideally zero due to the perfect orthogonality and it is showing the smallest value among the other filters.

The channel model is a Rayleigh fading channel with uncorrelated scattering (US) and a tapped-delay profile

$$
c(t, \tau)=\sum_{n=0}^{N c-1} c_{n}(t) \delta\left(\tau-\tau_{n}\right)
$$

Where $\tau_{n}$ is the $n^{\text {th }}$ delay and these delays are chosen to be fixed and equally spaced such that $\tau_{n}=30 n \mathrm{~ns}, N_{c}$ is the number of taps and is chosen that $N_{c}=21$ taps, this gives a maximum path delay $\tau_{\max }=600 \mathrm{~ns}$. The coefficients $c_{n}$ are complex Gaussian samples, each has a Jakes Doppler spectrum with an exponential power profile [15]

$$
S(\tau)=\left\{\begin{array}{cc}
e^{\tau / \sigma} / \sigma & \tau \geq 0 \\
0 & \tau<0
\end{array}\right.
$$

Where $\sigma$ is the root mean square (RMS) delay of the continuous delay profile in (32). The actual rms delay $\tau_{r m s}$ of the discrete profile in (31) does not equal to $\sigma$ due to the discrete and finite nature of (31), so, it is calculated through the simulation. For a detailed treatment of the discretized exponential channel profile, the reader may refer to [15]. It is well known that the $\tau_{\text {rms }}$ has the most impact on the system performance over $\tau_{\max }$ and even the power profile [18], so, the performance of the shaping filters will be analyzed against $\tau_{\text {rms }}$. Another aspect regarding the channel simulation is that the choice of the sampling intervals of the signal and the channel, which in many cases are chosen equally for performance evaluation purposes. In our case, they are unequal as stated earlier. In order to make valid analytical treatment, the channel model should be altered in order to fit the sampling interval of the signal, and this is accomplished by the transforming equation [18]

$$
d_{k}=\sum_{n=0}^{N_{c}-1} c_{n} \operatorname{sinc}\left(\tau_{n} / t_{s}-k\right) \quad-N_{d} \leq k \leq N_{d}
$$

Where $d_{k}$ is the new set of channel coefficients and $N_{d}$ is chosen such that coefficients beyond the index $N_{d}$ become insignificant. The simulation results are presented over a range of $\tau_{r m s}$ and for different values of Doppler frequency $f_{d}$ as shown in Fig. 3 through Fig. 6. The value of $\tau_{\text {rms }}$ is varied by varying the value of $\sigma$ in (32) while keeping the same channel profile. The comparison shows clearly the difference between different pulse shaping filter while the performance is the same against different channel parameters $\tau_{r m s}$ and $f_{d}$. 


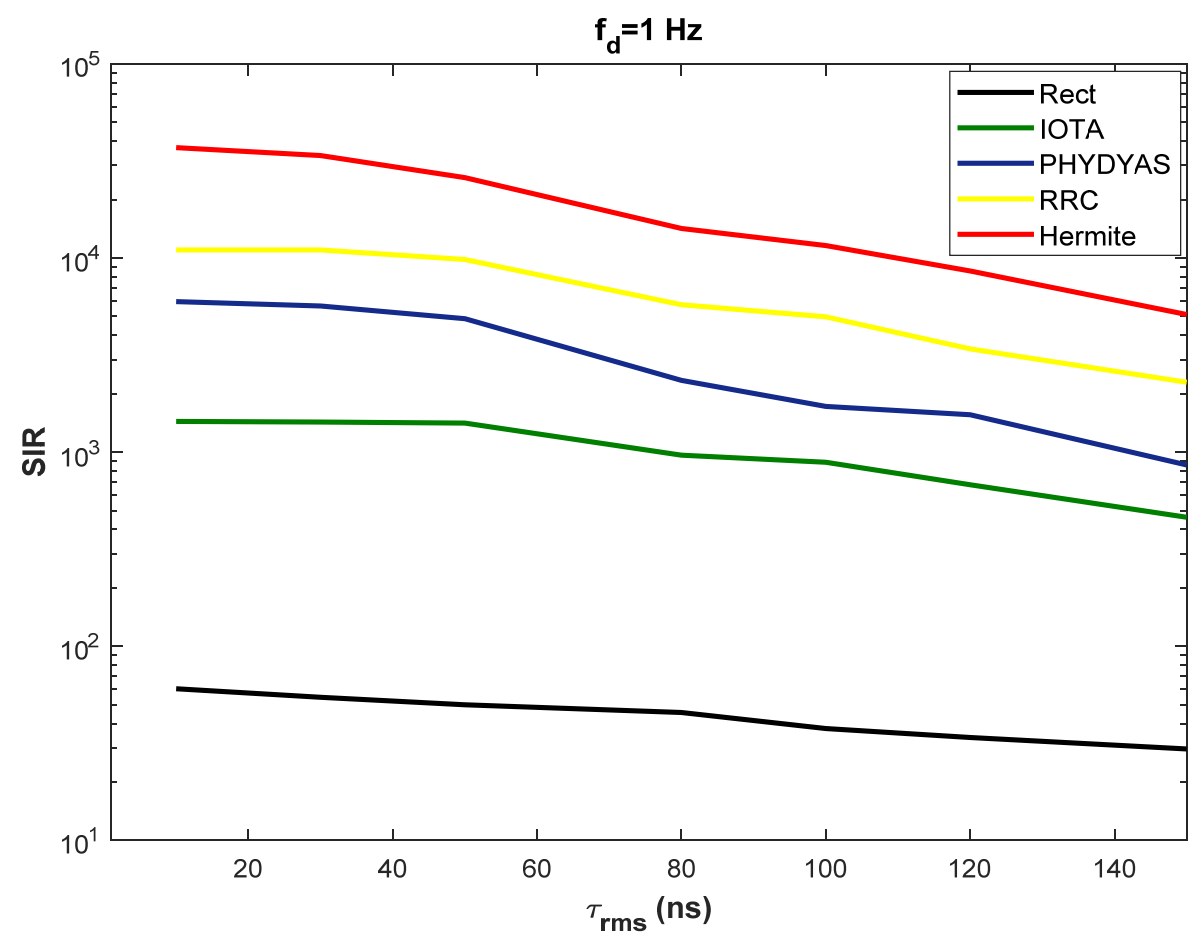

FIG. 3. SIR vs $\tau_{r m s}$ FOR DOPPLER FREQUENCY $f_{d}=1 \mathrm{~Hz}$.

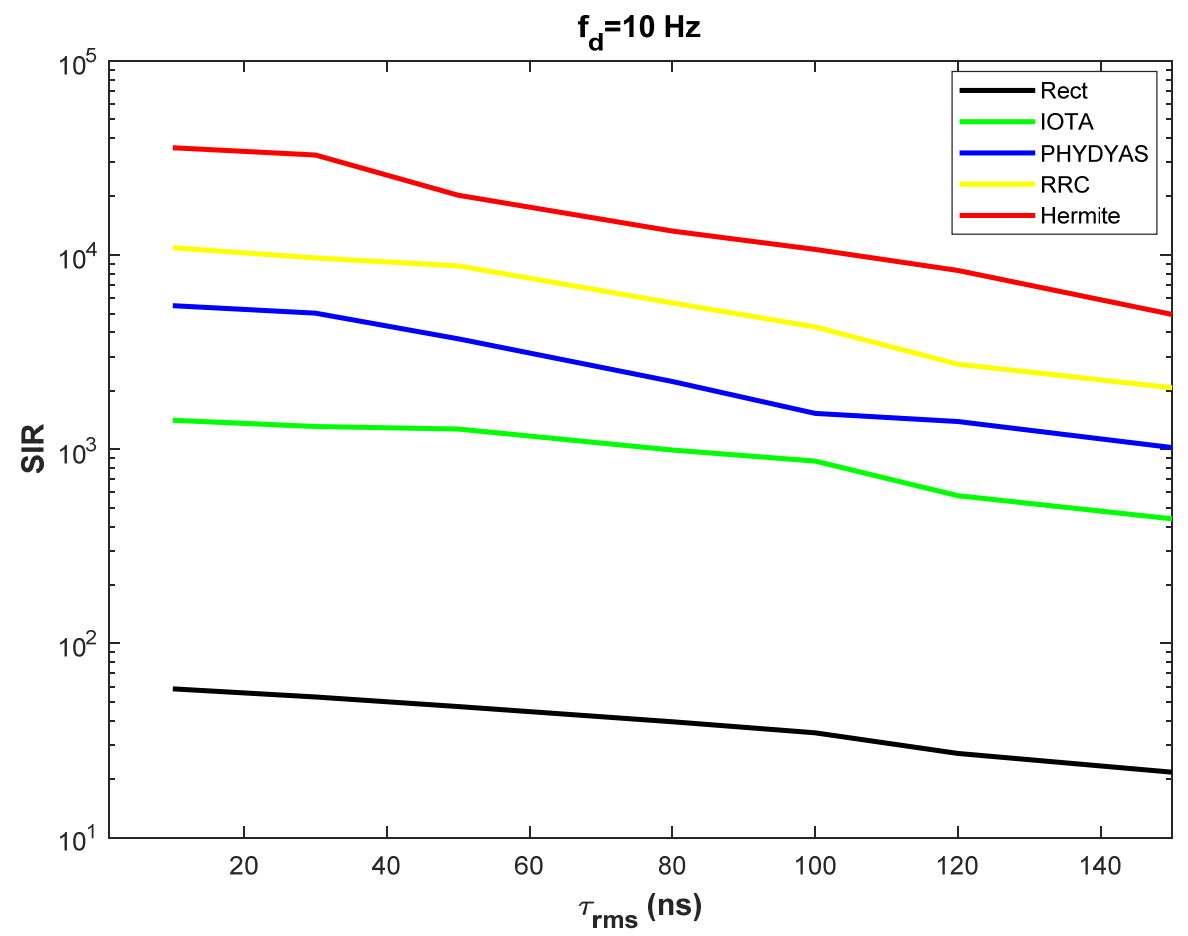

FIG. 4. SIR vs $\tau_{r m s}$ FOR DOPPLER FREQUENCY $f_{d}=10 \mathrm{~Hz}$. 


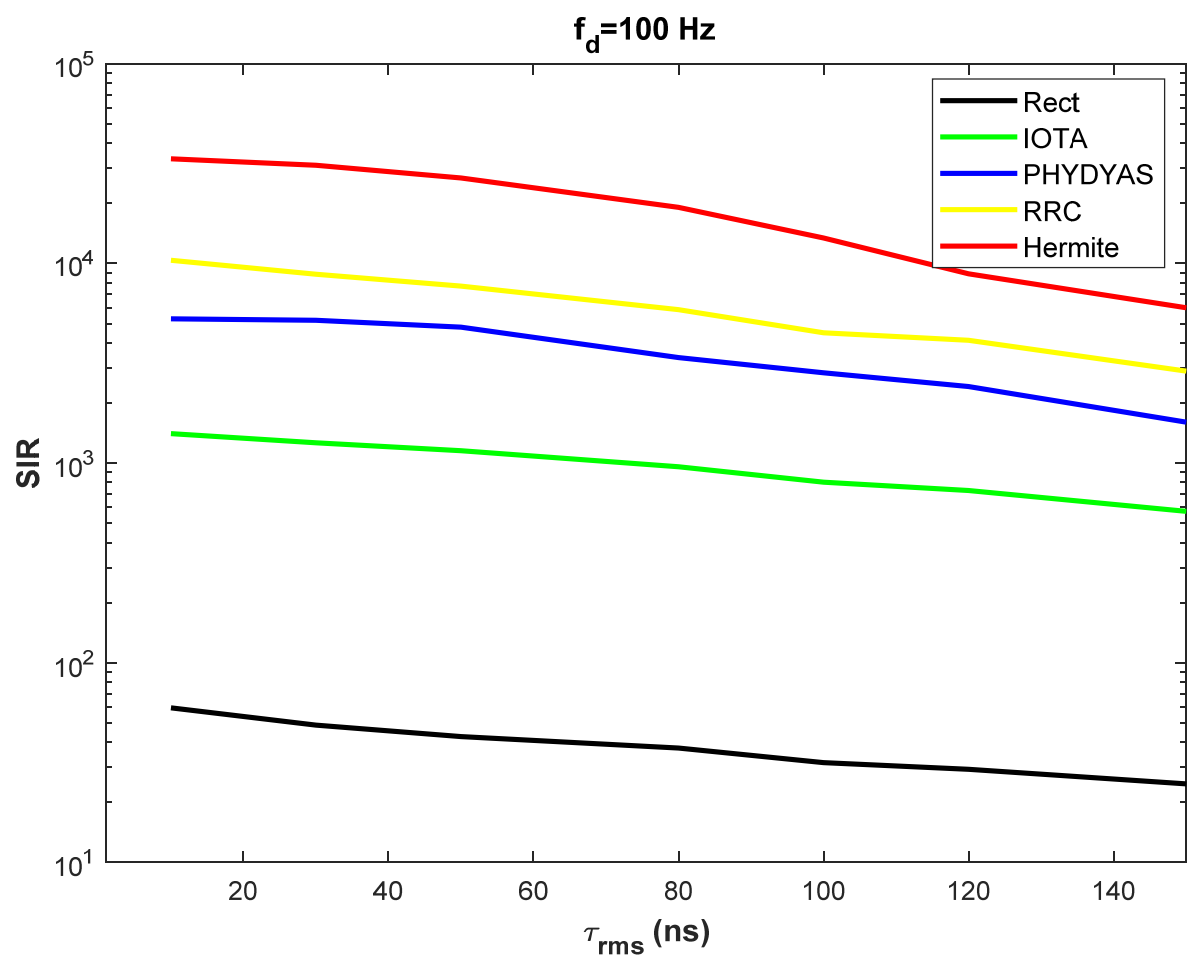

FIG. 5. SIR vs $\tau_{r m s}$ FOR DOPPLER FREQUENCY $f_{d}=100 \mathrm{~Hz}$.

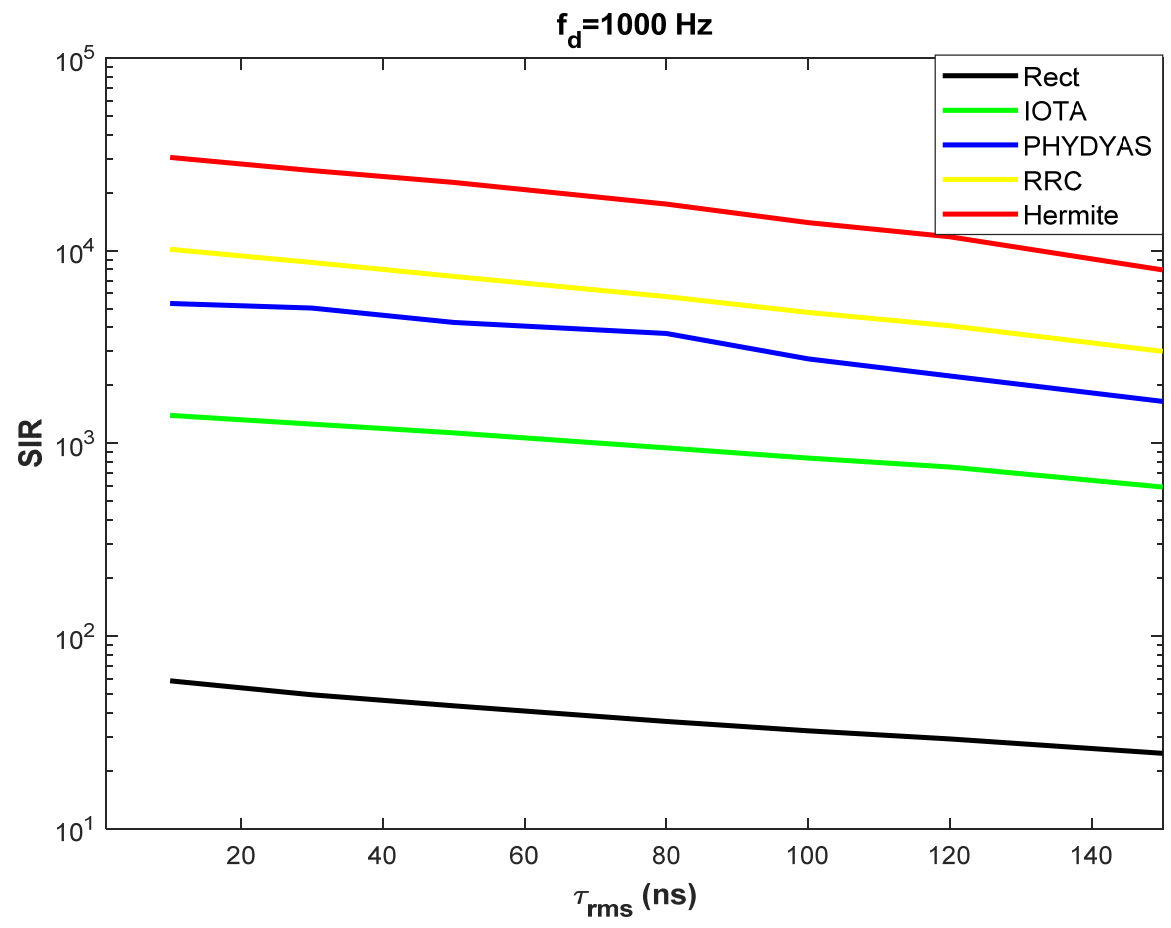

FIG. 6. SIR vs $\tau_{r m s}$ FOR DOPPLER FREQUENCY $f_{d}=1000 \mathrm{~Hz}$.

\section{CONCLUSION}

In this paper, the main types of pulse shaping filters used in FBMC are analyzed and compared for their orthogonality, localization, and performance against time-frequency dispersive channel for 
various values of time spreading and Doppler frequency. the filter with the best localization which is the Hermite function filter showed the best performance against the dispersive effect of the channel

\section{REFERENCES}

[1] R. Nissel and M. Rupp "OFDM and FBMC-OQAM in doubly-selective channels: calculating the bit error probability," IEEE Communications Letters, Volume. 21, Issue 6, June 2017.

[2] D. Gregoratti and X. Mestre, "Distortion analysis in OQAM/FBMC-based OFDMA" IEEE 16th International Workshop on Signal Processing Advances in Wireless Communications (SPAWC), Stockholm, 2015.

[3] Z. Zhao, M, Schellmann, X, Gong, Q, Wang, R, Böhnke and Y, Guo "Pulse shaping design for OFDM systems," EURASIP Journal on Wireless Communications and Networking, (2017) 2017:74

[4] M. Xu, J. Zhang, F. Lu, Y. Wang, D. Guidotti, and G. K. Chang, "Investigation of FBMC in mobile fronthaul networks for $5 \mathrm{~g}$ wireless with time-frequency modulation adaptation," Optical Fiber Communications Conference and Exhibition (OFC), California,

[5] B. Farhang-Boroujeny, "OFDM versus filter bank multicarrier," IEEE Sig. Proc. Mag., vol. 28, no. 3, pp. 92-112, May 2011.

[6] J. Du, and S. Signell "Time-frequency localization of pulse shaping filters in OFD/OQAM systems," 6th International Conference on Information, Communications \& Signal Processing, Dec. 2007, Singapore.

[7] H. G. Feichtinger and T. Strohmer, Gabor analysis and algorithms, Springer Science \& Business Media, 1998.

[8] J. Du, and S. Signell "Pulse Shape Adaptivity in OFDM/OQAM Systems," Internationa Conference of Advanced Infocomm Technology (ICAIT), Shen Zhen, China, July 2008.

[9] M. Bellanger, "FBMC physical layer: a primer," Tech. Rep. 06/2010, 2010.

[10] A. Şahin, 'İ. Güvenç, and H. Arslan, "A Survey on Prototype Filter Design for Filter Bank Based Multicarrier Communications," IEEE Communications Surveys \& Tutorials, Dec. 2013.

[11] J. Bazzi, P. Weitkemper, K. Kusume, A. Benjebbour, Y. Kishiyama "Design and Performance Tradeoffs of Alternative Multi-Carrier Waveforms for 5G,” in Proc. IEEE Globecom Workshops, San Diego, Dec. 2015, pp. 1-6.

[12] B. Farhang-Boroujeny, "A square-root Nyquist (M) filter design for digital communication systems," IEEE Trans. Sig. Proc., vol. 56, no. 5, pp. 2127-2132, May 2008.

[13] R. Haas and J.-C. Belfiore, “A time-frequency well-localized pulse for multiple carrier transmission, "Wireless Pers. Commun., vol. 5, pp. 1-18, 1997.

[14] M. Alard. "Construction of a multicarrier signal," Patent WO 96/35278, 1996.

[15] C. A. Gutierrez and M. Cabrera, "Issues of the Simulation of Wireless Channels with Exponential-Decay Power-Delay Profiles," 2005 IEEE 16th International Symposium on Personal, Indoor and Mobile Radio Communications, Berlin, Sept. 2005, 507-511.

[16] P. Siohan, C. Siclet, and N. Lacaille, "Analysis and design of OFDM/OQAM systems based on filterbank theory," IEEE Trans. Sig. Proc., vol. 50, no. 5, pp. 1170-1183, May 2002.

[17] B. Boashash, Time-Frequency Signal Analysis, and Processing: A Comprehensive Reference, Elsevier Science, 2nd ed., 2015.

[18] M. C. Jeruchim, P. Balaban, K. S. Shanmugan, Simulation of Communication Systems: Modeling, Methodology and Techniques, Kluwer Academic Publishers, 2nd ed., 2000. 